The Annals of Applied Probability

2002, Vol. 12, No. 4, 1174-1184

\title{
SMOOTH GENERATORS OF INTEGRAL STOCHASTIC ORDERS
}

\author{
By Michel Denuit AND AlfRed MÜlleR \\ Université Catholique de Louvain and University of Karlsruhe
}

\begin{abstract}
The purpose of this paper is to show that many integral stochastic orders have a generator consisting of infinitely differentiable functions. This especially holds for all stochastic orders with characterizations via difference operators. The usefulness of this result is demonstrated in two applications relating to stochastic ordering of multivariate normal distributions and ordering of random vectors in a random environment.
\end{abstract}

1. Introduction. Stochastic orders are binary relations defined on sets of probability distributions, which formally describe intuitive ideas such as "being larger," "being more variable" or "being more dependent." Such stochastic order relations are now used in many areas of probability and statistics. For a comprehensive treatment see, for example, Shaked and Shanthikumar (1994), Müller and Stoyan (2002) or Szekli (1995).

Many of these orders have characterizations as so-called integral stochastic orders, that is, there is a class $\mathcal{F}$ of measurable functions such that $P \leq \mathcal{F} Q$ holds if, and only if, $\int f d P \leq \int f d Q$ for all $f \in \mathcal{F}$. A general study of this type of order has been given in Müller (1997). Typically there are several different classes of functions which characterize a given order. There is always a largest one, called maximal generator. This has been characterized in Theorem 3.7 of Müller (1997). Such large generators are interesting for applications of the orders $\leq \mathcal{F}$. However, for verifying that an integral stochastic order holds it is desirable to have small generators. In some cases (like the usual stochastic order $\leq_{s t}$ on the real line) there are nice small generators which make it easy to check the order. For some other orders, however, no small generators are available. This especially applies to the multivariate case.

For technical reasons, it is often helpful to know that it is sufficient to check $\int f d P \leq \int f d Q$ for all $f \in \mathcal{F}$ which are sufficiently smooth. Indeed, differentiability of test functions is assumed here and there in proofs without justifying that this is allowed. Smoothness makes us able, for example, to use integration by parts.

It is the aim of this paper to show that for all well-known integral stochastic orders it is allowed to assume that the generator consists only of infinitely differentiable functions. We derive our main theoretical results in Section 2, where

Received October 2000; revised March 2002.

AMS 2000 subject classifications. 60E15, 62H05.

Key words and phrases. Integral stochastic orders, infinitely differentiable generator, difference operator, multivariate normal distribution, random environment. 
it is shown that provided $\leq \mathcal{F}$ is closed under convolution and weak convergence, its generator can be restricted to smooth functions. In Section 3 this will be applied to a variety of integral stochastic orderings, especially to all orders with a generator that can be characterized via difference operators. In Section 4, we show how these results can be used for the comparison of multivariate normal distributions and for the ordering of random vectors in a random environment.

2. Main result. Let us first fix the notation. We use the setting of Müller (1997). Specifically, let $(S, \mathcal{A})$ be an arbitrary measure space and let $b: S \rightarrow$ $[1, \infty)$ be a measurable function, called weight function. We consider the set $\mathscr{B}_{b}$ of $b$-bounded measurable functions $f: S \rightarrow \mathbb{R}$, for which

$$
\|f\|_{b}:=\sup _{s \in S} \frac{|f(s)|}{b(s)}<\infty .
$$

Then any set of functions $\mathcal{F} \subset \mathscr{B}_{b}$ defines an order relation $\leq_{\mathcal{F}}$ on $\mathbb{P}_{b}$, the set of all probability measures on $(S, \mathcal{A})$ with $\int b d P<\infty$. $\mathcal{R}_{\mathcal{F}}$ shall be the corresponding maximal generator, which according to Theorem 3.7 of Müller (1997) is the closed convex cone (in the corresponding weak topology) spanned by $\mathcal{F}$ and the constant functions. In the following $S$ will always be some Euclidean space $S=\mathbb{R}^{d}$ endowed with the Borel $\sigma$-algebra, and as usual $\mathcal{C}^{\infty}$ shall denote the set of all functions on $S$ that are infinitely differentiable.

We will show that in all relevant cases $\mathcal{R}_{\mathcal{F}} \cap \mathcal{C}^{\infty}$ is an alternative generator of $\mathcal{F}$. Typically this can be achieved by a two-step procedure as follows. First show that the generator can be restricted to continuous functions. Then find for an arbitrary continuous $f \in \mathcal{R}_{\mathcal{F}}$ a sequence $\left\{f_{n}, n \in \mathbb{N}\right\}$ of infinitely differentiable functions $f_{n} \in \mathcal{R}_{\mathcal{F}}$ such that

$$
\lim _{n \rightarrow \infty} \int f_{n} d P=\int f d P
$$

for all $P \in \mathbb{P}_{b}$. In order to obtain the $f_{n}$ 's, we resort to the following well-known construction. Let $g$ be an infinitely differentiable probability density with a compact support, and let $g_{n}(\mathbf{x})=n^{d} \cdot g(n \cdot \mathbf{x})$. Then it is well known that the sequence

$$
f_{n}(\mathbf{x})=f * g_{n}(\mathbf{x}):=\int f(\mathbf{x}-\mathbf{y}) g_{n}(\mathbf{y}) d \mathbf{y}
$$

satisfies (1); see, for example, Rudin (1987), Theorem 9.10. Thus it only remains to be shown that $f_{n} \in \mathcal{R}_{\mathcal{F}}$. This question, however, is related to the question whether or not $\leq \mathcal{F}$ is closed under convolution, which means that $P_{1} \leq_{\mathcal{F}} P_{2}$ implies $P_{1} * Q \leq_{\mathcal{F}} P_{2} * Q$ for all $Q \in \mathbb{P}_{b}$. In fact, we have the following general result.

THEOREM 2.1. Let $\leq \mathcal{F}$ be an integral stochastic order generated by a class $\mathcal{F}$ of continuous functions. If $\leq \mathcal{F}$ is closed under convolution then there is a generator $g$ of this order relation which only consists of infinitely differentiable functions. Especially, $g=\mathcal{R}_{\mathcal{F}} \cap \mathcal{C}^{\infty}$ is such a generator. 
PROOF. We can assume without loss of generality that $\mathcal{F}$ consists of all continuous functions in $\mathcal{R}_{\mathcal{F}}$. Since $\leq_{\mathcal{F}}$ is closed under convolution, it follows from Theorem 4.2(e) in Müller (1997) that $\mathcal{F}$ is invariant under translations, and therefore $f(\cdot-\mathbf{y}) \in \mathcal{F}$ for all $\mathbf{y}$. Thus Theorem 3.6 in Müller (1997) ensures that the function $f_{n}$ defined in (2) is in $\mathcal{R}_{\mathcal{F}}$. Now define $g=\mathcal{R}_{\mathcal{F}} \cap \mathcal{C}^{\infty}$. Since $g \subset \mathcal{R}_{\mathcal{F}}$ it is obvious that $P \leq_{\mathcal{F}} Q$ implies $P \leq_{g} Q$. On the other hand, we have just shown that $f_{n} \in g$ for all $f \in \mathcal{F}$. Hence $P \leq q Q$ implies $\int f_{n} d P \leq \int f_{n} d Q$ and by equation (1) $\int f d P \leq \int f d Q$ for all $f \in \mathcal{F}$. Therefore we also have that $P \leq_{g} Q$ implies $P \leq \mathcal{F} Q$; that is, $\leq \mathcal{F}$ and $\leq q$ are identical.

Recall that a stochastic order $\leq \mathcal{F}$ is said to be closed under weak convergence, if for weak convergent sequences $\left\{P_{n}, n \in \mathbb{N}\right\}$ and $\left\{Q_{n}, n \in \mathbb{N}\right\}$ converging to $P$ and $Q$, respectively,

$$
P_{n} \leq_{\mathcal{F}} Q_{n} \text { for all } n \in \mathbb{N} \text { implies } P \leq \mathcal{F} Q .
$$

In Theorem 4.2(f) of Müller (1997) it has been shown that an integral stochastic order is closed under weak convergence if, and only if, it has a generator consisting of bounded continuous functions. Hence we can derive the following corollary from Theorem 2.1.

COROLLARY 2.2. If $\leq_{\mathcal{F}}$ is closed under weak convergence and under convolution then it has a generator $g \subset \mathrm{C}^{\infty}$.

3. Stochastic orders defined by difference operators. A large number of stochastic orders with a generator can be characterized in terms of difference operators. Define for a function $f: \mathbb{R}^{d} \rightarrow \mathbb{R}, \mathbf{x} \in \mathbb{R}^{d}, i \in\{1, \ldots, d\}$ and $\varepsilon>0$ the difference operator $\Delta_{i}^{\varepsilon}$ as

$$
\Delta_{i}^{\varepsilon} f(\mathbf{x})=f\left(\mathbf{x}+\varepsilon \mathbf{e}_{i}\right)-f(\mathbf{x}),
$$

where $\mathbf{e}_{i}=(0, \ldots, 0,1,0, \ldots, 0)$ denotes the $i$ th unit vector. In case $d=1$ we simply write

$$
\Delta^{\varepsilon} f(x)=f(x+\varepsilon)-f(x) .
$$

For a tuple $\boldsymbol{\imath}=\left(i_{1}, \ldots, i_{k}\right)$ of numbers $i_{j} \in\{1, \ldots, d\}$ we define the generator $\mathcal{F}_{\boldsymbol{l}^{+}}$ which shall consist of all functions such that

$$
\Delta_{i_{1}}^{\varepsilon_{1}} \cdots \Delta_{i_{k}}^{\varepsilon_{k}} f(\mathbf{x}) \geq 0 \quad \text { for all } \mathbf{x} \in \mathbb{R}^{d}, \varepsilon_{1}, \ldots, \varepsilon_{k}>0 .
$$

and let $\mathcal{F}_{\boldsymbol{l}^{-}}$be the set of functions with

$$
\Delta_{i_{1}}^{\varepsilon_{1}} \cdots \Delta_{i_{k}}^{\varepsilon_{k}} f(\mathbf{x}) \leq 0 \quad \text { for all } \mathbf{x} \in \mathbb{R}^{d}, \varepsilon_{1}, \ldots, \varepsilon_{k}>0 .
$$

Given a set $\Im$ of such "signed indices" let

$$
\mathcal{F}_{\Im}=\bigcap_{\boldsymbol{l} \in \Im} \mathcal{F}_{\boldsymbol{l}} .
$$


Many integral stochastic orders that have been considered in the literature possess a generator of the form $\mathcal{F}_{\Im}$, as it can be seen from the following examples.

Let us start with univariate stochastic orders, that is, the case $d=1$. Denote by $\boldsymbol{u}_{k}=(1, \ldots, 1)$ the tuple consisting of $k$ repetitions of the number 1 . Notice that

$$
\Delta^{\varepsilon} f(x) \geq 0 \quad \text { for all } x \in \mathbb{R} \text { and all } \varepsilon>0
$$

if and only if $f$ is increasing. Hence $\mathcal{F}_{\boldsymbol{l}_{1}^{+}}$is the set of all increasing function and thus the generator of the (usual) stochastic order $\leq_{s t}$. Similarly,

$$
\Delta^{\varepsilon_{1}} \Delta^{\varepsilon_{2}} f(x) \geq 0 \quad \text { for all } x \in \mathbb{R} \text { and all } \varepsilon_{1}, \varepsilon_{2}>0
$$

if and only if $f$ is convex. Hence $\widetilde{F}_{\boldsymbol{t}_{2}^{+}}$generates the convex order $\leq_{c x}$, and taking the intersection yields that $\mathcal{F}_{\Im_{2}}$ with $\Im_{2}=\left\{\boldsymbol{\iota}_{1}^{+}, \boldsymbol{\iota}_{2}^{+}\right\}$generates the increasing convex order $\leq_{i c x}$. Similarly $\mathcal{F}_{\boldsymbol{t}_{2}^{-}}$generates the concave order $\leq_{c v}$, and the increasing concave order $\leq_{i c v}$ is obtained from $\mathfrak{\Im}_{2}^{-}=\left\{\boldsymbol{l}_{1}^{+}, \boldsymbol{l}_{2}^{-}\right\}$. Considering higher differences yields the so-called $s$-convex orderings considered by Denuit, Lefèvre and Shaked (1998). There the ordering generated by $\widetilde{F}_{\boldsymbol{t}_{s}^{+}}$is called $\leq_{s-c x}$ and the ordering $\leq_{s-i c x}$ has the generator $\mathcal{F}_{\Im_{s}}=\left\{\boldsymbol{l}_{1}^{+}, \ldots, \boldsymbol{l}_{s}^{+}\right\}$. Another ordering with such a characterization is the Laplace order $\leq_{L t}$ which is defined as follows. $X \leq_{L t} Y$ holds if $E e^{-t X} \geq E e^{-t Y}$ for all $t>0$. At first sight there seems not be a characterization of the kind described above. Reuter and Riedrich (1981), however, have shown that the maximal generator of Laplace order consists of all functions with

$$
\begin{aligned}
(-1)^{n+1} \Delta^{\varepsilon_{1}} \cdots \Delta^{\varepsilon_{n}} f(x) \geq 0 \quad & \text { for all } x \in \mathbb{R}, \varepsilon_{1}, \ldots, \varepsilon_{n}>0 \\
& \text { and all } n=1,2, \ldots .
\end{aligned}
$$

Thus it is generated by $\mathcal{F}_{\Im}$ with $\Im=\left\{\boldsymbol{\imath}_{1}^{+}, \boldsymbol{\iota}_{2}^{-}, \boldsymbol{\iota}_{3}^{+}, \ldots\right\}$.

There are also numerous multivariate stochastic orders that can be characterized in this way. Notice that a function $f: \mathbb{R}^{d} \rightarrow \mathbb{R}$ is increasing if and only if

$$
\Delta_{i}^{\varepsilon} f(\mathbf{x}) \geq 0 \quad \text { for all } \mathbf{x} \in \mathbb{R}^{d}, \varepsilon>0 \text { and } i=1, \ldots, d .
$$

Hence the multivariate version of $\leq_{s t}$ is generated by $\mathcal{F}_{\Im_{s t}}$ for $\mathfrak{I}_{s t}=\left\{i^{+}: i=\right.$ $1, \ldots, d\}$.

A function $f: \mathbb{R}^{d} \rightarrow \mathbb{R}$ is said to be supermodular if

$$
\Delta_{i}^{\varepsilon} \Delta_{j}^{\delta} f(\mathbf{x}) \geq 0 \quad \text { for all } \mathbf{x} \in \mathbb{R}^{d}, \varepsilon, \delta>0 \text { and } 1 \leq i<j \leq d .
$$

Properties and applications of the stochastic order generated by this class of functions have been considered by Bäuerle (1997), Shaked and Shanthikumar (1997) and Müller and Scarsini (2000), among others. It is easy to see that this supermodular order (denoted by $\leq_{s m}$ ) is generated by $\mathcal{F}_{\Im_{s m}}$ where $\mathfrak{\Im}_{s m}=\left\{(i, j)^{+}: 1 \leq i<\right.$ $j \leq d\}$. A similar concept is that of the directionally convex order $\leq d c x$ which has been shown to be useful, for example, in Bäuerle and Rolski (1998), Meester 
and Shanthikumar (1999) and Müller and Scarsini (2001). A function is called directionally convex if it is supermodular and, in addition, convex in each argument, when the others are held fixed. Hence it is generated by $\mathcal{F}_{\Im_{d c x}}$, where $\mathfrak{\Im}_{d c x}=\left\{(i, j)^{+}: i, j=1, \ldots, d\right\}$. Combining supermodularity respectively directional convexity with monotonicity yields the increasing supermodular order $\leq_{i s m}$ which is generated by $\mathcal{F}_{\Im_{s m}} \cap \mathcal{F}_{\Im_{s t}}$ and the increasing directional convex order $\leq_{i d c x}$ generated by $\widetilde{F}_{\Im_{d c x}} \cap \mathcal{F}_{\Im_{s t}}$.

Finally we want to mention the lower and upper orthant orders, defined as

$$
\begin{aligned}
\mathbf{X} \leq{ }_{u o} \mathbf{Y} & \text { if } P\left(X_{1}>x_{1}, \ldots, X_{d}>x_{d}\right) \leq P\left(Y_{1}>x_{1}, \ldots, Y_{d}>x_{d}\right) \\
& \text { for all } x_{1}, \ldots, x_{d}
\end{aligned}
$$

and

$$
\begin{aligned}
\mathbf{X} \leq_{l o} \mathbf{Y} & \text { if } P\left(X_{1} \leq x_{1}, \ldots, X_{d} \leq x_{d}\right) \geq P\left(Y_{1} \leq x_{1}, \ldots, Y_{d} \leq x_{d}\right) \\
& \text { for all } x_{1}, \ldots, x_{d} .
\end{aligned}
$$

These orders both have generators defined by difference operators, too. In fact the maximal generator of $\leq_{u o}$ is the set of all functions with

$$
\Delta_{i_{1}}^{\varepsilon_{1}} \cdots \Delta_{i_{k}}^{\varepsilon_{k}} f(\mathbf{x}) \geq 0 \quad \text { for all } \mathbf{x} \in \mathbb{R}^{d}, \varepsilon_{1}, \ldots, \varepsilon_{k}>0
$$

and all distinct $i_{1}, \ldots, i_{k}, 1 \leq k \leq d$. This result can be traced back to Rüschendorf (1980). Hence $\leq_{u o}$ is generated by $\mathcal{F}_{\Im_{u o}}$, where $\mathfrak{\Im}_{u o}=\left\{\left(i_{1}, \ldots, i_{k}\right)^{+}: i_{1}, \ldots, i_{k}\right.$ distinct, $1 \leq k \leq d\}$. A similar characterization is possible for $\leq_{l o}$ as it is generated by the class of all functions $f$ such that $f(-\mathbf{x})$ is in $\mathcal{F}_{\Im_{u o}}$.

Now we will show that for all these orderings Theorem 2.1 applies. First observe the following result.

THEOREM 3.1. Let $\mathfrak{\Im}$ be an arbitrary set of signed indices and let $\leq \mathcal{F}_{\mathfrak{Y}}$ be the stochastic order generated by the corresponding class $\mathcal{F}_{\Im}$ of functions. Then $\leq \mathcal{F}_{\mathfrak{Y}}$ is closed under convolution.

PROOF. It is obvious from the definition that for a fixed index $\boldsymbol{l}$ the sets $\mathcal{F}_{\boldsymbol{l}^{+}}$ and $\mathcal{F}_{\boldsymbol{l}^{-}}$are invariant under translations. Hence $\mathcal{F}_{\Im}$ as an intersection of such sets is again invariant under translations. Thus the assertion follows from Theorem 4.2(e) in Müller (1997).

Since all of the orders mentioned above are known to have generators consisting only of continuous functions, we can apply Theorem 2.1 to show that all these orders have a generator consisting of infinitely differentiable functions. The nice feature of the next theorem is that it means that we can just translate the conditions given in terms of differences into conditions given in terms of derivatives. 
Therefore, let us define for a given $\boldsymbol{\imath}=\left(i_{1}, \ldots, i_{k}\right)$ the set $\mathcal{F}_{\boldsymbol{t}^{+}}^{\infty}\left(\mathcal{F}_{\boldsymbol{t}^{-}}^{\infty}\right)$ as the set of all infinitely differentiable functions with the property that

$$
\frac{\partial^{k}}{\partial x_{i_{1}} \cdots \partial x_{i_{k}}} f(\mathbf{x}) \geq(\leq) 0 \quad \text { for all } \mathbf{x} \in \mathbb{R}^{d},
$$

and for a given set $\mathfrak{\Im}$ of such signed indices let

$$
\mathcal{F}_{\Im}^{\infty}=\bigcap_{\boldsymbol{i} \in \Im} \mathcal{F}_{\boldsymbol{I}}^{\infty} .
$$

We get the following result.

THEOREM 3.2. Let $\mathcal{F}_{\Im}$ be the generator of one of the stochastic orders $\leq_{s t}$, $\leq_{s-c x}, \leq_{s-i c x}, \leq_{s-c v}, \leq_{s-i c v}, \leq_{L t}, \leq_{s m}, \leq_{i s m}, \leq_{d c x}, \leq_{i d c x}, \leq_{u o}$ or $\leq_{l o}$. Then $\leq_{\mathcal{F}_{\mathfrak{F}}}$ is also generated by $\mathcal{F}_{\Im}^{\infty}$ which consists only of infinitely differentiable functions.

PROOF. In view of Theorem 2.1 we have to show that the mentioned orders are closed under convolution and that there is a generator consisting of continuous functions. The closure under convolution has been shown in general in Theorem 3.1. Hence it remains to be shown that there is always a generator consisting of continuous functions. For $\leq_{s t}$ this is known since Kamae, Krengel and O'Brien (1977). For the supermodular and increasing supermodular order this has been shown in Müller and Scarsini (2000). A similar proof is possible for the orthant order. In all other cases it is obvious that even the maximal generator contains only continuous functions.

We want to mention explicitly a few important examples that are contained in Theorem 3.2. Given two $d$-dimensional random vectors $\mathbf{X}$ and $\mathbf{Y}$ :

1. $\mathbf{X} \leq_{s m} \mathbf{Y}$ if, and only if, $E f(\mathbf{X}) \leq E f(\mathbf{Y})$ holds for all twice differentiable functions $f: \mathbb{R}^{d} \rightarrow \mathbb{R}$ satisfying $\partial^{2} / \partial x_{i} \partial x_{j} f(\mathbf{x}) \geq 0$ for all $\mathbf{x} \in \mathbb{R}^{d}$ and all $1 \leq i<j \leq d$.

2. $\mathbf{X} \leq_{i s m} \mathbf{Y}$ if, and only if, $E f(\mathbf{X}) \leq E f(\mathbf{Y})$ holds for all twice differentiable functions $f: \mathbb{R}^{d} \rightarrow \mathbb{R}$ satisfying $\partial / \partial x_{i} f(\mathbf{x}) \geq 0$ for all $\mathbf{x} \in \mathbb{R}^{d}$ and all $1 \leq i \leq d$ and $\partial^{2} / \partial x_{i} \partial x_{j} f(\mathbf{x}) \geq 0$ for all $\mathbf{x} \in \mathbb{R}^{d}$ and all $1 \leq i<j \leq d$.

3. $\mathbf{X} \leq_{d c x} \mathbf{Y}$ if, and only if, $E f(\mathbf{X}) \leq E f(\mathbf{Y})$ holds for all twice differentiable functions $f: \mathbb{R}^{d} \rightarrow \mathbb{R}$ satisfying $\partial^{2} / \partial x_{i} \partial x_{j} f(\mathbf{x}) \geq 0$ for all $\mathbf{x} \in \mathbb{R}^{d}$ and all $1 \leq i, j \leq d$.

4. $\mathbf{X} \leq i d c x \mathbf{Y}$ if, and only if, $E f(\mathbf{X}) \leq E f(\mathbf{Y})$ holds for all twice differentiable functions $f: \mathbb{R}^{d} \rightarrow \mathbb{R}$ satisfying $\partial / \partial x_{i} f(\mathbf{x}) \geq 0$ for $1 \leq i \leq d$ and $\partial^{2} / \partial x_{i} \partial x_{j} f(\mathbf{x}) \geq 0$ for all $\mathbf{x} \in \mathbb{R}^{d}$ and all $1 \leq i, j \leq d$.

At the end of this section we want to mention that there are also stochastic orders to which Theorem 2.1 applies though they cannot be characterized via difference operators. The most important examples are the multivariate versions 
of the convex and increasing convex orders. Specifically, $\mathbf{X}$ is said to precede $\mathbf{Y}$ in the multivariate convex order, written as $\mathbf{X} \leq_{c x} \mathbf{Y}$, if $E f(\mathbf{X}) \leq E f(\mathbf{Y})$ holds for any convex function $f: \mathbb{R}^{d} \rightarrow \mathbb{R}$, provided the expectations exist. In this case, Theorem 2.1 applies, since a translation of a convex function again is convex, and any convex function must be continuous. Hence $\mathbf{X} \leq_{c x} \mathbf{Y}$ holds if and only if $E f(\mathbf{X}) \leq E f(\mathbf{Y})$ holds for all twice differentiable functions $f: \mathbb{R}^{d} \rightarrow \mathbb{R}$ with a positive semidefinite Hesse matrix. The same applies to the multivariate increasing convex order, written as $\mathbf{X} \leq_{i c x} \mathbf{Y}$, which holds if $E f(\mathbf{X}) \leq E f(\mathbf{Y})$ holds for any nondecreasing and convex function $f: \mathbb{R}^{d} \rightarrow \mathbb{R}$, provided the expectations exist; in this case, Theorem 2.1 applies, too.

REMARK 3.3. A number of notions of positive dependence have been introduced in the literature in an effort to mathematically describe the property that "large values of the components of a random vector go together with large values of the other components." Many of these notions are defined by means of some comparison of the random vector $\mathbf{X}$ with its independent version $\mathbf{X}^{\perp}$ (which shall have the same marginals as $\mathbf{X}$, but independent components); we refer the interested reader to, for example, Szekli (1995) or Scarsini and Shaked (1996). For instance, the upper orthant dependence is defined as $\mathbf{X}^{\perp} \leq{ }_{u o} \mathbf{X}$, the lower orthant dependence as $\mathbf{X} \leq_{l o} \mathbf{X}^{\perp}$. All the results derived in this paper thus obviously apply to dependence notions defined with the help of integral stochastic orderings.

\section{Applications.}

4.1. Ordering Gaussian vectors. As a first application the results of Section 3 can be used to characterize stochastic ordering of multivariate normal distributions; such characterizations can be found in Müller (2001) and are based on the following identity. Let $\mathbf{X} \sim \mathcal{N}(\boldsymbol{\mu}, \boldsymbol{\Sigma})$ and $\mathbf{Y} \sim \mathcal{N}\left(\boldsymbol{\mu}^{\prime}, \boldsymbol{\Sigma}^{\prime}\right)$, and let $\phi_{\lambda}$ be the density of $\mathcal{N}\left(\lambda \boldsymbol{\mu}^{\prime}+(1-\lambda) \boldsymbol{\mu}, \lambda \boldsymbol{\Sigma}^{\prime}+(1-\lambda) \boldsymbol{\Sigma}\right), 0 \leq \lambda \leq 1$. Moreover, assume that $f: \mathbb{R}^{d} \rightarrow \mathbb{R}$ is twice continuously differentiable and satisfies some polynomial growth conditions. Then

$$
\begin{aligned}
E f(\mathbf{Y})-E f(\mathbf{X})=\int_{0}^{1} \int & \left(\left(\boldsymbol{\mu}^{\prime}-\boldsymbol{\mu}\right)^{T} \nabla f(\mathbf{x})+\frac{1}{2} \operatorname{tr}\left(\left(\boldsymbol{\Sigma}^{\prime}-\mathbf{\Sigma}\right) \mathbf{H}_{f}(\mathbf{x})\right)\right) \\
& \times \phi_{\lambda}(\mathbf{x}) d \mathbf{x} d \lambda,
\end{aligned}
$$

where $\nabla f$ and $H_{f}$ are the gradient and the Hessian of $f$, respectively, and $\operatorname{tr}(\mathbf{A})$ denotes the trace of the matrix $\mathbf{A}$.

This identity is then used to derive characterizations of the most important integral stochastic orders. In the proofs it is tacitly assumed that it is allowed to restrict the generators to twice differentiable functions. Thus Theorem 2.1 together with Section 3 fills a little gap in the proofs there. 
4.2. Ordering random vectors in a random environment. Consider the following model of a $d$-dimensional random vector $\mathbf{X}=\left(X_{1}, \ldots, X_{d}\right)$ depending on a random environment $\Theta=\left(\Theta_{1}, \ldots, \Theta_{d}\right)$ in the following fashion:

1. Component $X_{i}$ depends only on $\Theta_{i}$; that is, given $\boldsymbol{\Theta}=\boldsymbol{\theta}$, the distribution of $X_{i}$ depends only on $\theta_{i}$ and is independent of $\theta_{k}$ for $k \neq i$.

2. The components of $\mathbf{X}$ are conditionally independent; that is, given $\boldsymbol{\Theta}=\boldsymbol{\theta}$ the random variables $X_{1}, \ldots, X_{d}$ are independent.

Unconditionally, however, there may be dependence in the vector $\mathbf{X}=$ $\left(X_{1}, \ldots, X_{d}\right)$ induced by the dependence structure of $\boldsymbol{\Theta}=\left(\Theta_{1}, \ldots, \Theta_{d}\right)$. It is an interesting question how the distribution of $\boldsymbol{\Theta}$ affects the distribution of $\mathbf{X}$, especially how the dependence structure of $\mathbf{X}$ depends on the one of $\boldsymbol{\Theta}$. In the language of stochastic orderings this question can be stated as follows: Let $\boldsymbol{\Theta}$ and $\boldsymbol{\Theta}^{\prime}$ be two random environments, $\mathbf{X}$ and $\mathbf{X}^{\prime}$ the corresponding random observations with the same conditional marginal distribution functions

$$
F_{i}(x \mid \theta)=P\left(X_{i} \leq x \mid \Theta_{i}=\theta\right)=P\left(X_{i}^{\prime} \leq x \mid \Theta_{i}^{\prime}=\theta\right)
$$

and let $\leq_{\mathcal{F}}$ be a stochastic order of interest. Under what conditions does $\boldsymbol{\Theta} \leq_{\mathcal{F}} \boldsymbol{\Theta}^{\prime}$ imply $\mathbf{X} \leq \mathcal{F} \mathbf{X}^{\prime}$ ?

Various problems of this type can be found in the recent literature. Bäuerle [(1997), Lemma 3.4] shows such a result for the supermodular order in the context of queueing systems in a random environment. Her proof is based on a coupling argument. In the context of synchronized stochastic systems, $\mathrm{Li}$ and $\mathrm{Xu}$ (2000) study so-called separable Markov chains which have transition kernels of this type. In their Theorem 2.7 they prove results of the above-mentioned nature for supermodular order and orthant orders. In actuarial sciences similar results have recently been given by $\mathrm{Hu}$ and Pan (1999), Denuit, Genest and Marceau (2002) and Lillo, Pellerey, Semeraro and Shaked (2000).

It is not surprising that the answer to the problem mentioned above is related to stochastic monotonicity and stochastic convexity conditions on $F_{i}(x \mid \theta)$. Recall that $X_{i}$ is said to be stochastically increasing (resp. convex) in $\Theta_{i}$ if the function

$$
\theta \mapsto E\left[f\left(X_{i}\right) \mid \Theta_{i}=\theta\right]
$$

is increasing (resp. convex), whenever $f$ is an increasing (resp. convex) function. In the proof below we will use the well-known facts that $X_{i}$ is stochastically increasing in $\Theta_{i}$ if, and only if,

$$
\theta \mapsto P\left(X_{i}>x \mid \Theta_{i}=\theta\right)=1-F_{i}(x \mid \theta)
$$

is increasing for all $x$, and that it is stochastically convex if and only if $\theta \mapsto$ $E\left[X_{i} \mid \Theta_{i}=\theta\right]$ is linear and

$$
\theta \mapsto \pi_{i}(x \mid \theta)=E\left[\left(X_{i}-x\right)_{+} \mid \Theta_{i}=\theta\right]=\int_{x}^{\infty}\left[1-F_{i}(y \mid \theta)\right] d y
$$

is convex; see, for example, Denuit and Lefèvre [(2001), Proposition 1] for a proof of the latter claim. 
THEOREM 4.1. (a) Assume that $X_{i}$ is stochastically increasing in $\Theta_{i}$ for all $i=1,2, \ldots, d$ and let $\preceq$ be one of the stochastic orders $\leq_{s t}, \leq_{s m}, \leq_{i s m}, \leq_{u o}$ and $\leq_{l o}$. Then the following implication holds:

$$
\boldsymbol{\Theta} \preceq \boldsymbol{\Lambda} \Rightarrow \mathbf{X} \preceq \mathbf{Y} .
$$

(b) If $X_{i}$ is stochastically convex in $\Theta_{i}$ for all $i=1,2, \ldots, d$ then

$$
\boldsymbol{\Theta} \leq_{d c x} \boldsymbol{\Lambda} \Rightarrow \mathbf{X} \leq_{d c x} \mathbf{Y} .
$$

(c) If $X_{i}$ is stochastically increasing and stochastically convex in $\Theta_{i}$ for all $i=1,2, \ldots, d$ then

$$
\boldsymbol{\Theta} \leq_{i d c x} \boldsymbol{\Lambda} \Rightarrow \mathbf{X} \leq_{i d c x} \mathbf{Y} .
$$

PROOF. Let us write

$$
E f(\mathbf{X})=\int E[f(\mathbf{X}) \mid \boldsymbol{\Theta}=\boldsymbol{\theta}] F_{\boldsymbol{\Theta}}(d \boldsymbol{\theta})=E f^{*}(\boldsymbol{\Theta}),
$$

where

$$
f^{*}(\boldsymbol{\theta})=\int f(\mathbf{x}) \bigotimes_{k=1}^{d} F_{k}\left(d x_{k} \mid \theta_{k}\right)
$$

Here $\otimes$ denotes the product measure. According to the results derived in Section 3 it is sufficient to consider infinitely differentiable functions $f$. Moreover, we assume without loss of generality that $f$ has bounded derivatives of all orders. For the supermodular order it has been shown in Müller and Scarsini (2000) that this is sufficient and for the directional convex order in Müller and Scarsini (2001). For all other orders in the theorem this is obvious.

Now the differentiability of $f$ enables us to perform partial integrations to get the following identities:

$$
\Delta_{i}^{\varepsilon} f^{*}(\boldsymbol{\theta})=\int \frac{\partial}{\partial x_{i}} f(\mathbf{x})\left\{F_{i}\left(x_{i} \mid \theta_{i}\right)-F_{i}\left(x_{i} \mid \theta_{i}+\varepsilon\right)\right\} \bigotimes_{k \neq i} F_{k}\left(d x_{k} \mid \theta_{k}\right) d x_{i}
$$

for all $i=1, \ldots, d$,

$$
\begin{aligned}
\Delta_{i}^{\varepsilon} \Delta_{j}^{\delta} f^{*}(\boldsymbol{\theta}) & \\
=\int \frac{\partial^{2}}{\partial x_{i} \partial x_{j}} & f(\mathbf{x})\left\{F_{i}\left(x_{i} \mid \theta_{i}\right)-F_{i}\left(x_{i} \mid \theta_{i}+\varepsilon\right)\right\} \\
& \times\left\{F_{j}\left(x_{j} \mid \theta_{j}\right)-F_{j}\left(x_{j} \mid \theta_{j}+\delta\right)\right\} \bigotimes_{k \neq i, j} F_{k}\left(d x_{k} \mid \theta_{k}\right) d x_{i} d x_{j}
\end{aligned}
$$

for all $i, j=1, \ldots, d$ with $i \neq j$, and

$$
\begin{aligned}
\Delta_{1}^{\varepsilon_{1}} & \cdots \Delta_{d}^{\varepsilon_{d}} f^{*}(\boldsymbol{\theta}) \\
& =\int \frac{\partial^{d}}{\partial x_{1} \cdots \partial x_{d}} f(\mathbf{x}) \prod_{i=1}^{d}\left(F_{i}\left(x_{i} \mid \theta_{i}\right)-F_{i}\left(x_{i} \mid \theta_{i}+\varepsilon_{i}\right)\right) d x_{1} \cdots d x_{d} .
\end{aligned}
$$


From these equations part (a) immediately follows.

If $X_{i}$ is stochastically convex in $\Theta_{i}$ then $E\left[X_{i} \mid \Theta_{i}=\theta\right]$ is linear in $\theta$; that is, $E\left[X_{i} \mid \Theta_{i}=\theta\right]=a_{i}+b_{i} \theta$ for all $i=1, \ldots, d$. Hence

$$
\begin{aligned}
& \lim _{x_{i} \rightarrow-\infty}\left\{\pi_{i}\left(x_{i} \mid \theta_{i}+2 \varepsilon\right)-2 \pi_{i}\left(x_{i} \mid \theta_{i}+\varepsilon\right)+\pi_{i}\left(x_{i} \mid \theta_{i}\right)\right\} \\
& \quad=E\left[X_{i} \mid \Theta_{i}=\theta_{i}+2 \varepsilon\right]-2 E\left[X_{i} \mid \Theta_{i}=\theta_{i}+\varepsilon\right]+E\left[X_{i} \mid \Theta_{i}=\theta_{i}\right]=0 .
\end{aligned}
$$

Therefore applying another integration by parts to (6) yields

$$
\begin{gathered}
\Delta_{i}^{\varepsilon} \Delta_{i}^{\varepsilon} f^{*}(\boldsymbol{\theta})=\int \frac{\partial^{2}}{\partial x_{i}^{2}} f(\mathbf{x})\left\{\pi_{i}\left(x_{i} \mid \theta_{i}+2 \varepsilon\right)-2 \pi_{i}\left(x_{i} \mid \theta_{i}+\varepsilon\right)+\pi_{i}\left(x_{i} \mid \theta_{i}\right)\right\} \\
\bigotimes_{k \neq i} F_{k}\left(d x_{k} \mid \theta_{k}\right) d x_{i} .
\end{gathered}
$$

From this identity parts (b) and (c) follow immediately, too.

Many parametric families of distributions fulfill the assumptions of Theorem 4.1; see, for example, Chapter 6 of Shaked and Shanthikumar (1994).

\section{REFERENCES}

BäUerle, N. (1997). Monotonicity results for MR/GI/1 queues. J. Appl. Probab. 34 514-524.

BÄUerle, N. and Rolski, T. (1998). A monotonicity result for the work-load in Markovmodulated queues. J. Appl. Probab. 35 741-747.

Denuit, M., Genest, C. and Marceau, E. (2002). A criterion for the stochastic ordering of random sums. Scand. Actuar. J. 2002 3-16.

Denuit, M. and LefÈvre, C. (2001). Stochastic $s$-(increasing) convexity. Generalized Convexity and Generalized Monotonicity. Lecture Notes in Econom. and Math. Systems $\mathbf{5 0 2}$ 167-182. Springer, New York.

Denuit, M., LefÈvre, C. and Shaked, M. (1998). The $s$-convex orders among real random variables, with applications. Math. Inequal. Appl. 1 585-613.

Hu, T. and PAN, X. (1999). Preservation of multivariate dependence under multivariate claim models. Insurance Math. Econom. 25 171-179.

Kamae, T., Krengel, U. and O'Brien, G. L. (1977). Stochastic inequalities on partially ordered spaces. Ann. Probab. 5 899-912.

LI, H. and XU, S. (2000). The dependence structure and bounds of correlated parallel queues and their applications to synchronized stochastic systems. J. Appl. Probab. 37 1020-1043.

Lillo, R. E., Pellerey, F., Semeraro, P. and Shaked M. (2000). On the preservation of the supermodular order under multivariate claim models. Preprint, Univ. Tucson, AZ.

Meester, L. E. and Shanthikumar, J. G. (1999). Stochastic convexity on general space. Math. Oper. Res. 24 472-494.

Müller, A. (1997). Stochastic orders generated by integrals: A unified study. Adv. Appl. Probab. 29 414-428.

MÜller, A. (2001). Stochastic ordering of multivariate normal distributions. Ann. Inst. Statist. Math. 53 567-575.

Müller, A. and SCARSINI, M. (2000). Some remarks on the supermodular order. J. Multivariate Anal. 61 107-119. 
MÜller, A. and SCARSINI, M. (2001). Stochastic comparison of random vectors with a common copula. Math. Oper. Res. 26 723-740.

MÜLler, A. and StoyAn, D. (2002). Comparison Methods for Stochastic Models and Risks. Wiley, Chichester.

REUTER, H. and RIEDRICH, T. (1981). On maximal sets of functions compatible with a partial ordering for distribution functions. Math. Operationsforsch. Statist. Ser. Optim. 12597 605.

Rudin, W. (1987). Real and Complex Analysis. McGraw-Hill, New York.

RÜSCHENDORF L. (1980). Inequalities for the expectation of $\Delta$-monotone functions. Z. Wahrsch. Verw. Gebiete 54 341-349.

SCARsini, M. and ShaKed, M. (1996). Positive dependence orders: A survey. In Athens Conference on Applied Probability and Time Series I: Applied Probability (C. C. Heyde et al., eds) 70-91. Springer, New York.

Shaked, M. and Shanthikumar, J. G. (1994). Stochastic Orders and their Applications. Academic Press, London.

Shaked, M. and Shanthikumar, J. G. (1997). Supermodular stochastic orders and positive dependence of random vectors. J. Multivariate Anal. 61 86-101.

SzekLI, R. (1995). Stochastic Ordering and Dependence in Applied Probability. Lecture Notes in Statist. 97. Springer, New York.

INSTITUT DE STATISTIQUE

UNIVERSITÉ CATHOLIQUE DE LOUVAIN

20 VOIE DU ROMAN PAYS

B-1348 LOUVAIN-LA-NEUVE

BELGIUM

E-MAIL: denuit@stat.ucl.ac.be
INSTITUT FÜR WIRTSCHAFTSTHEORIE

UND OPERATIONS RESEARCH

UNIVERSITÄT KARLSRUHE, GEB. 20.21

D-76128 KARLSRUHE

GERMANY

E-MAIL: mueller@wior.uni-karlsruhe.de 